\title{
Corela
}

Cognition, représentation, langage

HS-1 1 | 2012

RJC Cotexte, contexte, situation

\section{Situations d'occurrence des emplois du futur simple français sur la base d'un corpus économique de presse}

\section{Alida Maria Silletti}

\section{CpenEdition}

Journals

Édition électronique

URL : https://journals.openedition.org/corela/2597

DOI : $10.4000 /$ corela.2597

ISSN : 1638-573X

Éditeur

Cercle linguistique du Centre et de l'Ouest - CerLICO

Référence électronique

Alida Maria Silletti, «Situations d'occurrence des emplois du futur simple français sur la base d'un corpus économique de presse », Corela [En ligne], HS-11 | 2012, mis en ligne le 31 janvier 2012, consulté le 21 septembre 2021. URL : http://journals.openedition.org/corela/2597 ; DOI : https:// doi.org/10.4000/corela.2597

Ce document a été généré automatiquement le 21 septembre 2021.

\section{c.) (i) (2)}

Corela - cognition, représentation, langage est mis à disposition selon les termes de la licence Creative Commons Attribution - Pas d'Utilisation Commerciale - Partage dans les Mêmes Conditions 4.0 International. 


\title{
Situations d'occurrence des emplois du futur simple français sur la base d'un corpus économique de presse
}

\author{
Alida Maria Silletti
}

\section{Introduction}

1 Cette contribution ${ }^{1}$ aborde les valeurs sémantiques que le futur simple peut assumer dans certaines conditions d'énonciation. En particulier, nous avançons l'hypothèse que l'interprétation des emplois d'une forme verbale découle de la forme verbale elle-même, mais également de son origine et du cotexte et du contexte qui l'entourent. La partie empirique de notre travail s'appuie sur un corpusde presse, tiré de la rubrique «Economie» du Figaro, qui nous servira pour relever les différents emplois du futur simple français. De même, nous étudierons la manière dont le contexte et le cotexte influencent le décodage de ces lectures spécifiques du futur.

2 Par conséquent, d'abord nous mettrons en relief certaines difficultés posées par l'analyse $\mathrm{du}$ futur en français. Ensuite, nous présenterons notre corpus et la répartition des emplois du futur simple, ce qui nous permettra d'opérer des distinctions, justifiées par la présence ou par l'absence d'une série d'indices - la personne verbale, la diathèse, la typologie des phrases où les futurs apparaissent, les alliances «verbo-adverbiales». En effet, ces éléments peuvent modifier, sous certaines conditions, le sens de l'énoncé et la valeur du futur. Pour finir, le repérage de la fréquence et des fonctions remplies par le futur simple nous aidera, d'une part, à préciser son rôle à l'intérieur du discours de vulgarisation économique, de l'autre, à comprendre jusqu'à quel point une situation énonciative « restreinte» et/ou « étendue » (Kerbrat-Orecchioni, 1980) peut interférer dans les différentes interprétations du futur. 


\section{Genèse du futur simple français}

3 Parmi les différentes formes verbales, le cas du futur est tout à fait particulier. En effet, " "future" is not simply a division of linear time that follows chronologically upon the present. Aspect and above all modality are involved in reference to the future " (Fleischman, 1982 : 21). En plus, la catégorie verbale du futur a fait son apparition sans aucun doute après les catégories du passé, sous la forme de prétérit, et du présent (Bybee et Dahl, 1989).

Quant aux langues romanes, déjà en latin, l'affirmation et la définition de la forme verbale qualifiée de « futur » posaient des difficultés remarquables. Dans cette langue, des périphrases modales exprimant le futur, chargées de nuances affectives différentes, ont graduellement remplacé les futurs flexionnels unis du latin ${ }^{2}$. Tout cela est attesté par la tendance de certaines langues, dont le français, à manifester le futur verbal au moyen de deux éléments, l'un marquant le signifié lexical (le verbe à l'infinitif), l'autre le signifié grammatical (le présent d'un verbe auxiliaire ou modal), d'où la construction cantare habeo (III siècle après J.-C.)du latin vulgaire, ensuite chanterai. Au début, cette périphrase aurait gardé son sens propre de volonté sous forme de contrainte. C'est pourquoi elle était plus forte que le futur synthétique latin, qui en revanche indiquait une "futurité " plutôt générique. Seulement plus tard elle s'est vidée de son contenu affectif pour acquérir un sens neutre, en devenant ainsi la seule forme de futur verbal.

Il ressort qu'il est difficile de bien délimiter le futur simple, étant donné que la pluralité de ses valeurs résulte de sa genèse. De fait, la référence à cette forme verbale n'implique pas toujours le renvoi à la tranche temporelle du futur, et, plus généralement, à l'avenir. Donc, le futur français se démarque par une certaine instabilité, qui se répercute sur la variété de ses emplois.

\section{Présentation du corpus}

Notre corpus se compose de 2328 textes (643066 mots) tirés de la rubrique «Economie » du Figaro pendant la période $1^{\mathrm{er}}$ janvier - 28 février 2007. Les textes téléchargés correspondent à la version numérique au format pdf du quotidien imprimé.

7 En ce qui concerne la nature des textes de notre corpus, nous nous sommes partiellement appuyée sur le classement des textes proposé par Eggs (1998). Toutefois, nous reconnaissons que "[l]es locuteurs disposent d'une foule de termes pour catégoriser l'immense variété des textes qui sont produits dans une société [...] » (Maingueneau, 1998: 45). Ainsi, nos textes de spécialité, avec une fonction principalement informative, ont-ils été répartis en articles journalistiques; reportages (textes qui dépassent les 100 mots, qui portent sur un sujet spécifique d'actualité et où le lieu de rédaction est précisé); interviews; commentaires (textes d'opinion); indices boursiers (commentaires aux cotations boursières); flashes (textes qui ne dépassent pas les 100 mots); titres (titres ou première partie de textes qui figurent à la une de l'économie et qui sont développés dans les pages qui suivent) ; schémas récapitulatifs et questions des lecteurs : 


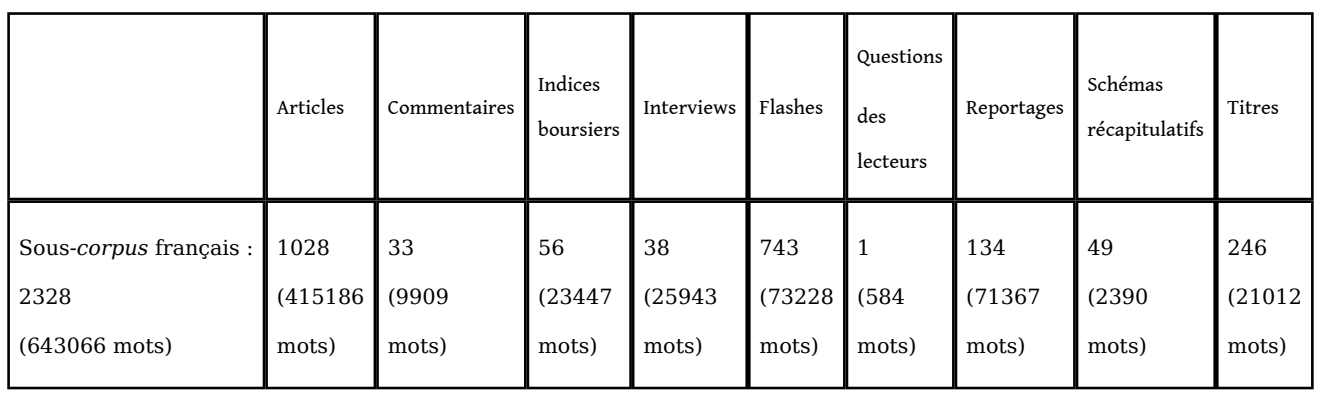

Tableau 1 - Répartition des textes et nombre de mots figurant dans les typologies textuelles du corpus

\section{Cadre méthodologique}

Pour le décodage des emplois du futur simple français nous nous appuyons sur l'importance revêtue par la situation d'énonciation. Dans tout type d'interaction, il est possible de distinguer des critères de nature « externe » et de nature « interne » (KerbratOrecchioni, 1990). Les premiers ont trait aux différentes composantes du contexte et sont par conséquent plus généraux, les seconds se réfèrent aux propriétés linguistiques et discursives du genre en question. Or, dans cette perspective, aussi bien le contexte que le genre peuvent être définis sur la base de ces deux critères, là où une "face interne », le fonctionnement linguistique, est opposée à une "face externe", les pratiques contextuelles. Autrement dit, Charaudeau et Maingueneau (2002), qui reprennent la distinction de Kerbrat-Orecchioni (1980) entre approche " étendue " et approche " restreinte ", soutiennent que l'une " a pour but de décrire les relations qui se tissent entre l'énoncé et les différents éléments constitutifs du cadre énonciatif ", l'autre "recherche les procédés linguistiques (shifters, modalisateurs, termes évaluatifs, etc.) par lesquels le locuteur imprime sa marque à l'énoncé, s'inscrit dans le message (implicitement ou explicitement) et se situe par rapport à lui (problème de la "distance énonciative ») (Charaudeau et Maingueneau, 2002 : 229).

9 Tout événement de communication est donc associé à des critères externes (la situation de discours, dont le lieu de la communication, la nature de ses participants, son/ses but/s, le type de canal, le degré de formalité de l'échange communicationnel), tout comme à des critères internes, car cette activité s'identifie au-delà des événements qui la composent. Les critères internes sont des éléments spécifiques qui découlent du matériel linguistique et de l'organisation du discours, dont la typologie de connecteurs utilisés, l'organisation des énoncés produits, l'utilisation des déictiques, l'emploi des temps verbaux. Il est évident que les mêmes critères internes et externes peuvent être appliqués tant à l'écrit qu'à l'oral, où de légères modifications peuvent intervenir ; pour la présente étude nous nous limiterons à l'écrit.

10 L'examen du futur simple s'inscrit alors en premier lieu parmi les critères internes de l'activité mise en place. Cependant, il n'est pas possible d'opérer une analyse exhaustive sans prendre en compte la situation de discours où le type d'activité a lieu. 


\section{Analyse qualitative des données}

11 Les valeurs du futur simple vont être analysées d'un point de vue quantitatif (logiciel AntConc3.2.1, version 2007) et qualitatif.

12 L'examen qualitatif s'aligne partiellement à Rocci (2005). Dans son volume consacré à la modalité épistémique, le linguiste analyse le fonctionnement des verbes modaux et du futur simple (principalement en italien, mais également en français) en discours à partir de l'hypothèse de l'interface entre sémantique et argumentation. En détail, dans son chapitre consacré au futur simple (le $7^{\text {ème}}$ ), il propose un vaste éventail d'emplois auxquels cette forme verbale peut se prêter d'après certains indices discursifs. L'émetteur du texte revêt une importance incontournable, d'où la focalisation sur sa subjectivité et sur les participants à la communication. Dans le cas du discours de vulgarisation économique, les participants sont représentés par le journaliste, un interviewé (pour les entretiens), et le public des lecteurs potentiels du quotidien. Quand on songe au discours économique, on y associe généralement le langage formalisé, des spécialistes, mais le niveau de vulgarisation économique - qui a le mérite de formuler les concepts de manière accessible aux non-initiés - ne doit pas être sous-estimé. Le renvoi à l'avenir et à l'idée de prévision y est fortement représentée grâce à une volonté généralisée de communiquer aux épargnants/ consommateurs les tendances des marchés et d'orienter leurs choix en termes d'investissements.

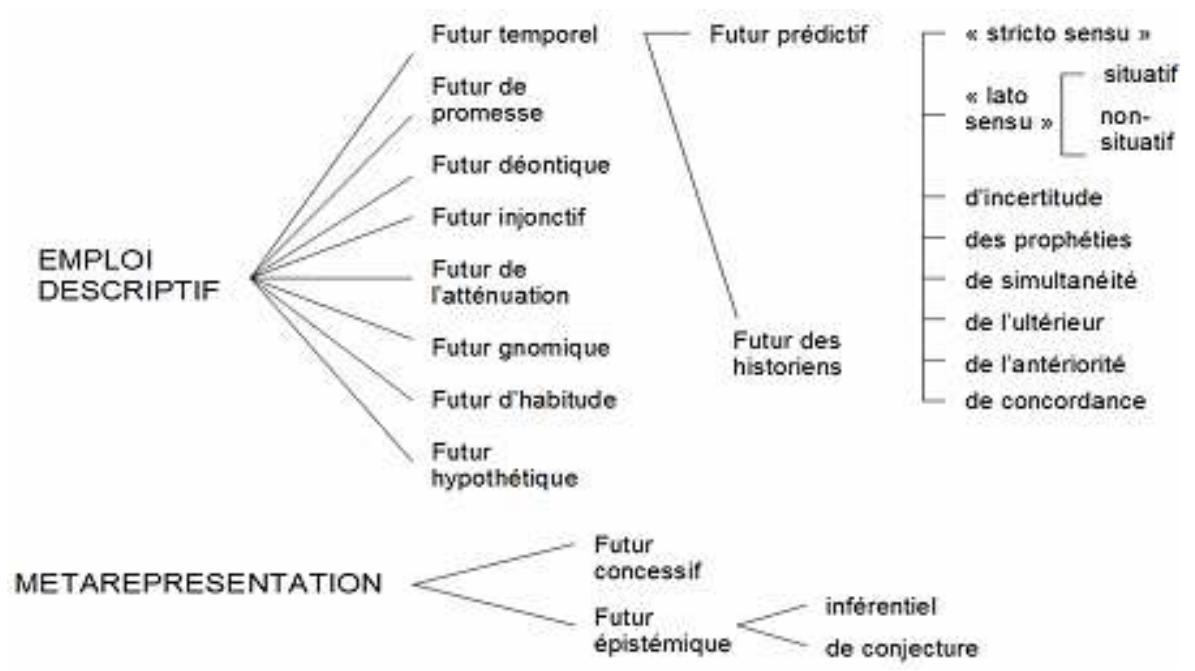

Figure 1 - Répartition des emplois du futur

Selon Rocci (2005), les emplois du futur ont été distingués en emploi descriptif (valeur temporelle au sens strict du futur) et métareprésentation (valeur modale du futur).

Dans le but de rechercher les relations que les emplois du futur tissent avec la situation d'énonciation (le discours de vulgarisation économique), quatre éléments vont être analysés: la personne verbale; la typologie de verbe et la diathèse; la typologie de phrase ; les alliances « verbo-adverbiales ». Nous n'allons traiter que des emplois les plus significatifs et de ceux qui figurent dans notre corpus. 


\subsection{Critère 1 : la personne verbale}

15 Nous partageons la considération de Celle (1997), qui affirme que «[i]l est d'ailleurs significatif que le futur ne soit pas analysé en fonction du sujet dans les grammaires françaises et dans les études qui lui sont consacrées [...] ; toutefois, dès lors qu'on analyse les emplois dits modaux du futur, ce critère intervient » (Celle, 1997: 63). En effet, la personne verbale joue un rôle fondamental pour déceler les emplois du futur, surtout si des nuances modales entrent en jeu dans l'interprétation du futur. Tel est le cas de la valeur la plus strictement déictique du futur - l'emploi prédictif -, où la personne verbale utilisée est négligeable. Au contraire, au sein de cinq autres emplois (futur des historiens ; futur déontique; futur injonctif; futur de promesse; futur épistémique), la personne verbale est essentielle.

Le futur des historiens fait percevoir l'événement comme appartenant à l'actualité du locuteur. Cependant, le moment de l'événement est reculé dans le temps. En outre, des références temporelles passées et l'utilisation exclusive de la $3^{\text {ème }}$ personne caractérisent cet emploi. 15 exemples figurent dans notre corpus (13 au singulier, 2 au pluriel) :

(1) En octobre 2003, Jean-Pierre Raffarin appelle Michel Boyon à Matignon, pour donner un deuxième souffle à son action, avant une année électorale qui se révélera catastrophique pour la majorité. (lf24-01-113).

17 L'emploi déontique concerne à notre avis deux propriétés primordiales du futur : l'origine $\mathrm{du}$ futur à partir de la périphrase latine cantare habeo, marquant l'obligation, et l'expression d'un événement à venir, assortie de fortes nuances modales. On relève donc un détachement partiel du renvoi à l'avenir au sens strict. Cet emploi figure surtout en présence de contraintes ou de règles qui émanent d'une autorité et qui pèsent sur ses destinataires. Les $3^{\text {èmes }}$ personnes (8 cas au singulier, 9 au pluriel) sont les seules répertoriées, dans des phrases pour la plupart au passif :

(2) Les directeurs des écoles de la fonction publique (instituts régionaux d'administration, écoles nationales des douanes, de l'administration pénitentiaire, de la magistrature, des impôts, de la police, École des officiers de gendarmerie, ENA...) et les préfets seront chargés d'informer les lycéens et les étudiants de ces concours. (lf15-02-17).

18 L'emploi injonctif présente des affinités avec l'emploi déontique à propos de la manifestation de l'injonction et/ou de l'imposition d'un ordre, mais il s'en distingue par rapport à la personne verbale. En effet, le sujet qui est la source de l'injonction doit figurer aux $2^{\text {èmes }}$ personnes. Toutefois, un seul exemple apparaît dans notre corpus, au pluriel, où le futur est accompagné du modal devoir. Ce résultat s'explique par les contextes propres au futur injonctif, généralement le discours direct et l'oral (à titre d'exemple, les reproches des parents envers leurs enfants pour leur imposer un ordre) :

(3) CETTE ANNÉE, c'est le 31 mai au plus tard que vous devrez renvoyer votre déclaration de revenus. Une échéance qui s'inscrit dans un environnement politique particulier. (lf20-01-33).

19 L'emploi de promesse porte sur la prise en charge énonciative de la part du sujet parlant vis-à-vis du procès énoncé. Celui-ci est responsable de son énonciation et s'engage à tenir la parole donnée; d'où l'usage des $1^{\text {ères }}$ personnes ( 6 occurrences au singulier, 23 au pluriel), dans notre corpus réservées aux interviews ou à des citations intégrées aux textes. Dans ces cas, le sujet parlant est explicitement impliqué dans le contenu de l'énonciation et il présente une forte volonté psychologique : 
(4) Dans les mois qui viennent, nous annoncerons le rachat d'une entreprise dont

le positionnement est stratégiquement très intéressant pour notre groupe ", promet le PDG de Belvédère. (lf16-01-37). d'enonciation " interne » représentée, d'une part, par une phrase où figure un marqueur de l'opposition, d'autre part, par un adverbial modulateur/baliseur de vérité. Même si aucune restriction de personne et de voix verbales ne touche à cet emploi du futur, les 8 occurrences répertoriées dans notre corpus figurent à la 3ème personne du singulier et à la voix active. En outre, elles présentent la même structure : les marques de l'opposition même si (forme explicite), même en (forme implicite) ou mais, suivies ou précédées de l'adverbe baliseur de vérité certes :

(7) Cette méthode permettra certes de financer ces nouvelles dépenses sans accroître le déficit public. Mais elle ne favorisera pas - au moins à court terme - le 
désendettement qui implique d'alléger le montant global de la dépense plutôt que

d'en modifier les équilibres. (lf15-02-13). théoriquement toujours figurer si le verbe en admet l'existence.

\subsection{Critère 3 : la typologie de phrases}

L'emploi d'atténuation (Imbs, 1968) et le futur de promesse partagent la participation du sujet parlant au contenu de son énonciation. Toutefois, si dans le futur de promesse le sujet parlant s'engage lui-même dans l'énonciation, à travers le futur d'atténuation il prend les distances de ses déclarations. Dans le premier cas la participation du sujet parlant est maximale, alors que dans l'autre cas le locuteur nuance sa pensée pour ne pas être trop direct. L'atténuation au futur se manifeste dans la plupart de nos exemples dans des formules signalées par des verbes à la voix active (dans 2 cas seulement à la voix réflexive/pronominale) qui visent à attirer l'attention ou à fournir des explications :

(8) On regrettera, entre parenthèses, que l'enquête de l'Insee décrivant par tranches de 10 minutes la vie de 15441 personnes remonte à 1999 et n'ait pas été refaite depuis la généralisation des 35 heures. (lf15-01-30).

Dans l'emploi de promesse, l'engagement du locuteur et sa prise en charge énonciative dénotent en revanche une volonté de maintenir les accords établis, tout comme de rassurer, à travers l'émission d'une sorte de promesse, les destinataires auxquels on s'adresse. Les verbes employés aux $1^{\text {ères }}$ personnes, pour la plupart à la voix active et au futur (sans des verbes modaux), indiquent l'élan vers un but et la volonté de l'atteindre. Outre être et avoir, qui sont les verbes les plus utilisés, nous avons repéré 3 cas de tenir, normalement employé dans la structure tenir des engagements (ex. 4), et 2 cas d'atteindre (ex. 5).

L'utilisation de la voix passive ne marque pas des traits particuliers parmi les emplois $\mathrm{du}$ futur. A notre avis, étant donné ses fortes connotations modales, le futur déontique aurait dû présenter un nombre remarquable d'occurrences au passif. Cependant, notre corpus montre 19 occurrences de futur déontique, dont seul $26 \%$ au passif. Le passif représente, à notre avis, l'expression d'une prise de distance nette par rapport au verbe même. Cela est confirmé par l'impossibilité (où le complément d'agent fait défaut) de connaitre ou de pouvoir relever le sujet responsable du procès. Par conséquent, cette voix du verbe peut figurer au futur et marquer l'obligation, au cas où le contexte en permettrait l'interprétation. Cette situation se vérifie en présence du futur déontique employé au passif (surtout en présence de devoir au futur au sens propre) :

(9) Cette nomination devra ensuite être entérinée par décret du chef de l'État, d'ici

à deux semaines. (lf27-02-39).

En ce qui concerne, enfin, la voix réflexive/pronominale, ses occurrences sont non seulement rares, mais ne montrent pas de contextes particuliers d'occurrence : elle peut

D’après la répartition des phrases en simples, principales et subordonnées (complétives ; relatives; circonstancielles) (Riegel, Pellat, Rioul : 1994), les types de phrases les plus fréquents dans le corpus sont les phrases principales (soit, qui régissent des subordonnées) et les phrases simples (à savoir, faute de rapport de subordination) (2093 occurrences, 74\%), suivies des subordonnées relatives (415 occurrences, 15\%), des complétives (235 occurrences, $8 \%$ ) et des circonstancielles (79 occurrences). Dans le but de présenter des tendances générales d'occurrence, nous nous concentrerons sur les types de phrases les plus fréquents dans notre corpus. 
31 Plusieurs emplois figurent dans des phrases simples, principales ou complétives (objectives ou interrogatives indirectes). Dans le cas de l'emploi prédictif «stricto sensu ${ }^{4}$ ", privilégié dans les contextes où le sens de prévision est dominant, les prévisions sont émises surtout à l'intérieur de phrases simples, principales ou subordonnées complétives objectives (dans ce dernier cas si le journaliste propose le point de vue de sujets impliqués dans la situation analysée) :

(10) Selon le Réseau du transport d'électricité, la France sera sous-capacitaire dès 2009-2010, particulièrement en électricité de pointe. (lf04-01-19) (phrase simple);

(11) Le président d'Alcan estime que la croissance de la demande mondiale d'aluminium permettra d'absorber rapidement l'excédent. (lf01-02-28) (phrase subordonnée complétive objective).

L'emploi prédictif «lato sensu » situatif5, comme son appellation même en témoigne, concerne des procès énoncés au futur, mais qui découlent de choix préalablement opérés. A ce propos, une indication temporelle précise, dans la plupart des cas une date, met le procès en contexte. Ainsi, le procès, même si au futur, est-il présenté comme définitif, presque dépourvu de l'incertitude qui est le propre du futur. Etant donné le caractère d'annonce de cet emploi, ses occurrences figurent surtout à l'intérieur de phrases simples :

(12) Les représentants du personnel se sont abstenus de donner un avis sur le plan de sauvegarde de l'emploi. Les lettres de licenciement partiront le 15 mars. (lf21-02-27).

Contrairement aux deux emplois préalables, la lecture d'incertitude du futur porte sur l'incertitude et sur l'impossibilité de savoir a priori si l'événement envisagé se vérifiera. Ce sont des indices « internes » qui permettent de relever cet emploi : des marques lexicales de doute (noms, adjectifs ou adverbes), mais surtout le discours rapporté sous forme de phrases subordonnées complétives interrogatives indirectes (21 exemples dans par rapport aux 37 occurrences totales):

(13) Reste à savoir comment et dans quels délais une industrie sonnée par la concurrence et plombée par ses retraites concrétisera ce nouvel effort qu'elle veut s'imposer. (lf09-01-33).

Quatre autres emplois prédictifs sont assujettis à l'enchaînement des phrases et aux relations temporelles qui sous-tendent les rapports phrastiques: les emplois de simultanéité, d'antériorité, de postérieurité et de concordance ${ }^{6}$. Les futurs d'antériorité et de concordance ne figurent, dans nos exemples, que dans des subordonnées circonstancielles temporelles de situation. En revanche, l'expression de la simultanéité et de la postérieurité dans le futur peuvent être également véhiculées par d'autres éléments dans la phrase, d'où des phrases simples :

(14) Ce marché représentera 500 millions d'euros de revenus (hors publicité) en

2013 et dépassera le milliard quatre ans plus tard. La bonne vieille télévision analogique va basculer en télévision numérique entre le 31 mars 2008 et fin 2011. (lf05-01-24) (futur prédictif de l'ultérieur).

L'emploi hypothétique concerne presque uniquement des phrases principales. La subordonnée hypothétique (protase), est très souvent introduite par si, plus rarement par d'autres structures similaires; l'apodose est généralement une principale, parfois une subordonnée complétive infinitive ou une subordonnée relative. Voilà un exemple d'apodose comme phrase principale (parmi les 133 répertoriés) :

(15) Si elle acquiert Nations Energy, elle contrôlera 28 \% de notre production. »

(lfo3-01-10). 

proprement modaux du futur, soit la «métareprésentation». Des adverbiaux épistémiques de l'atténuation et de la certitude, du type certes ou bien sûr, figurent généralement à côté de futurs concessifs (ex. 7), tandis que des adverbiaux épistémiques de la probabilité ou de la possibilité concourent souvent à la distinction des emplois épistémiques inférentiel et de conjecture (ex. 6).

\subsection{Indices « externes » des emplois du futur}

Les indices externes concernent, par rapport à notre corpus, l'emploi prédictif des prophéties, l'emploi des historiens, l'emploi déontique et l'emploi épistémique. Dans le cas de l'emploi prédictif des prophéties ${ }^{7}$, le sens de prévision est traité non seulement en 
termes de validation $\mathrm{du}$ procès dans l'avenir, mais aussi de rapprochement ou d'éloignement temporel par rapport au moment de l'énonciation, ainsi que de degré d'« acceptabilité » du contenu propositionnel. En effet, nous relevons des prédictions à moyen/long terme, presque visionnaires, mais aussi des situations prophétiques, parfois paradoxales, dont la réalisation est difficile, voire absurde et non envisageable. Cette sensation est perçue à partir de nos connaissances préalables du monde, d'indices « externes » :

(19) Des nouvelles applications offertes sur Internet par Google, par exemple, permettent d'imaginer qu'un jour les consommateurs se passeront des systèmes d'exploitation Windows et de sa nouvelle version Vista, ou même de logiciels bureautiques comme Office. (lf30-01-13).

L'emploi des historiens fait également référence au bagage de connaissances partagées par les interlocuteurs. En effet, il est évident que la référence temporelle porte sur le passé et que ce futur a un caractère fictif (ex. 1).

futur déontique est interprété sur la base des critères « internes » 1 et 2 , mais auss d'indices lexicaux et de la situation énonciative. De ce fait, cette valeur est le propre de la langue du droit (Bertinetto, 1986) ; toutefois, elle peut figurer dans des contextes semispécialisés, tels que notre corpus (ex. 2 et 9).

Enfin, l'emploi épistémique s'identifie à partir des critères «internes » 1, 2 et 4, mais également de la situation énonciative toute entière. L'expression d'une probabilité (emploi épistémique inférentiel) ou d'une possibilité (emploi épistémique de conjecture) sont en effet le résultat de procès préalables plus ou moins connus par le sujet parlant. L'ex. 20 fait supposer que des raisons valables sous-tendent les affirmations du journaliste, à travers l'expression d'une inférence soulignée par sans doute :

(20) Ce pourrait d'ailleurs être, au final, une très belle opération pour le numéro un mondial de la sidérurgie. Ce dernier cédera sans doute d'autres usines, vraisemblablement moins performantes que celle de Dofasco. Sur un plan financier, l'opération sera moins juteuse que prévue, mais sur un plan industriel, le groupe devrait être gagnant. (lf11-01-42).

Les connaissances préalables décroissent en revanche au fur et à mesure que l'on s'éloigne du domaine de la probabilité et que l'on entre dans le domaine de la possibilité :

(21) Le dollar aura-t-il un nouvel accès de faiblesse ? (lf04-01-8).

\section{Conclusions}

Cette étude a été focalisée sur certains indices « internes » et « externes » des emplois du futur simple français. Nous avons montré qu'il est rare qu'un seul facteur influence le décodage d'un emploi particulier du futur, mais que généralement plusieurs facteurs entrent en jeu. Il est possible que plusieurs emplois présentent les mêmes critères distinctifs, mais, dans ces cas, d'autres éléments interviennent pour décoder la valeur spécifique du futur. Toutefois, même si notre classement des emplois du futur peut apparaître rigoureux, la pratique nous montre que les différentes interprétations du futur se superposent et que leurs traits de distinction présentent des barrières très floues. Il s'ensuit que la seule manière pour éviter des confusions consiste à bien délimiter les bornes. 
Sur la base de nos données et de nos résultats, nous attribuons un rôle essentiel aux indices « internes » - le cotexte - et « externes » - le contexte - pour déceler les emplois du futur. Nous avons à ce propos dressé un tableau qui résume nos considérations :

\begin{tabular}{|c|c|c|c|c|c|c|c|}
\hline & Emplors & Fréquence & CRITERE 1 & CRITERE ? & CRITERE 3 & CRITERE 4 & $\begin{array}{c}\text { MUDICES } \\
\text { \&XXTERNES } \\
*\end{array}$ \\
\hline \multirow{16}{*}{ 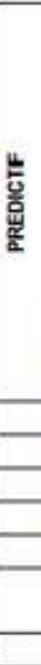 } & estreto sensu s & 746 & NON & NON & $\begin{array}{l}\text { PRWCSIMPUCOMP } \\
\text { LOBS }\end{array}$ & oui & NON \\
\hline & $\begin{array}{l}\text { ¿ lato sensu } \\
\text { stuatit }\end{array}$ & 289 & NON & NON & SIMPLES & oul & NON \\
\hline & $\begin{array}{l}\text { " lato sensu } \\
\text { non-stuatr }\end{array}$ & 1308 & NON & NON & NON & NON & NON \\
\hline & Des prophéties & 37 & NON & NON & HON & NON & OU1 \\
\hline & dincertiude & 37 & NON & NON & TNTERR IND & NON & NON \\
\hline & dantenorté & $T$ & NON & NON & TEMP & OU1 & NON \\
\hline & de futerieur & 3 & NON & NON & PRNCISIMPL & ou & NON \\
\hline & De simutraneté & 10 & NON & NON & TEMP & OU & NON \\
\hline & De concordance & 18 & NON & MON & TEMP & NON & MON \\
\hline & Des historiens & 15 & Oण & NON & Non & NON & OUI \\
\hline & De promesse & 56 & 001 & NON & आMPUCOUPL OBJ & NON & NON \\
\hline & Deontave & 19 & कण & oण & mon & NON & OUI \\
\hline & Thjoncit & 1 & Oण & Oण & NOH & NON & NON \\
\hline & Derattenuation & 9 & NON & कण & Non & NON & Non \\
\hline & Hypothétque & 141 & NON & NON & PRWIC & NON & NON \\
\hline & Concessif & 8 & NON & NON & PRWCSIMPL & oण & NON \\
\hline \multirow{2}{*}{$\frac{\text { 岁 }}{\frac{0}{\frac{5}{5}}}$} & oinference & 58 & oul & NON & NON & oul & oul \\
\hline & de conjecture & 55 & ovi & NON & NON & OUI & OUI \\
\hline
\end{tabular}

Tableau 2 - Fréquence et critères d'occurrence des emplois du futur simple dans le corpus

51 Les emplois du futur simple que nous venons de présenter sont extrêmement variés non seulement dans les définitions proposées, mais aussi par rapport à leur fréquence et aux éléments qui en permettent des distinctions ponctuelles à l'intérieur du corpus.

Nous tenons à souligner le caractère partiel de cette étude, étant donné que notre approche qualitative aux données du corpus a été essentiellement énonciative. En outre, notre corpus rend uniquement compte d'une section du quotidien, dont l'étude, pour la plupart morpho-syntaxique, a été consacrée au futur simple. Des recherches à venir pourraient donc mieux creuser le domaine de la «futurité », se concentrer également sur l'examen du présent de l'indicatif, mais aussi relever la manière dont le futur simple est traité dans d'autres sections du quotidien et/ou d'autres types de corpus.

\section{BIBLIOGRAPHIE}

Bertinetto P.M., Tempo, aspetto e azione nel verbo italiano, 1986, Florence : Accademia della Crusca

Bybee, J.L, Dahl, Ö, « The creation of tense and aspect systems in the languages of the world ", Studies in Language, 1989, 13-1:51-103 
Celle, A., Etude contrastive du futur français et de ses réalisations en anglais, 1997, Paris : Ophrys

Celle, A., " The French future tense and English will as markers of epistemic modality ", Languages in Contrast, 2004-2005, 5: $2: 181-218$

Charaudeau, P., Maingueneau, D. (dir.) Dictionnaire d'analyse du discours, 200, Paris : Seuil

Dahl, Ö., « The grammar of future time reference in European languages », dans Dahl, Ö. (dir.), Tense and Aspect in the Languages of Europe, 2000, Berlin/New York : Mouton : 309-328

Dendale P., «Le futur conjectural versus devoir épistémique: différences de valeurs et de restrictions d'emploi ", Le Français Moderne,2001, 69 (1) : 1-20

Eggs, E., Maîtrise du français et économie, 1998, Paris : Editions LIRIS

Fleishman, S., The future in thought and language. Diachronic Evidence from Romance, 1982, Cambridge : Cambridge University Press

Imbs P., L'emploi des temps verbaux en français moderne, 1968, Paris : Klincksieck

Kerbrat-Orecchioni, C., L'énonciation. De la subjectivité dans le langage, 1980, Paris : Armand Colin

Kerbrat-Orecchioni, C., Les interactions verbales, Tome I, 1990, Paris : Armand Colin

Kerbrat-Orecchioni, C., Les interactions verbales, Tome III, 1994, Paris : Armand Colin

Maingueneau, D., Analyser les textes de communication, 1998, Paris : Dunod

Riegel, M., Pellat, J.-C., Rioul, R., Grammaire méthodique du français, 1994, Paris : PUF

Rocci, A., La modalità epistemica tra semantica e argomentazione, 2005, Milan : Pubblicazioni dell'I.S.U. Università Cattolica

Silletti, A.M., La notion de «futurité » et sa réalisation morpho-syntaxique dans le discours de vulgarisation français/italien/anglais, 2009, thèse de doctorat non publiée, Università degli Studi di Modena e Reggio Emilia

Valesio, P., « La genesi del futuro romanzo »,Lingua e stile, 4, 1968 : 405-412

\section{NOTES}

1. Cette étude tire son origine de la thèse de doctorat La notion de «futurité » et sa réalisation morpho-syntaxique dans le discours de vulgarisation économique français/italien/anglais. Cependant, de nouveaux apports figurent en ce qui concerne la définition des indices « internes » et « externes » dans l'interprétation du futur.

2. Nous renvoyons à Valesio (1968) et à Fleischman (1982).

3. Le sigle à la fin des exemples est à lire de la manière suivante : If Le Figaro, suivi du jour, du mois et de la numérotation du texte par journée.
4. Notre nomenclature.
5. Ibid.
6. Ibid.
7. Ibid. 


\section{RÉSUMÉS}

Cette étude examine les conditions d'énonciation qui sous-tendent les valeurs sémantiques du futur simple français. Nous analyserons certains éléments cotextuels et contextuels qui peuvent avoir une influence sur la situation énonciative (Kerbrat-Orecchioni, 2002). L'exploration des emplois du futur portera sur un corpus de 2328 textes - 643236 mots - tirés de la section de l'économie du Figaro (1er janvier - 28 février 2007). Puisque la langue de l'économie concerne, par définition, des prévisions, notre but consistera, d'abord, à vérifier quels emplois sont censés se prêter à l'expression de la prévision. Ensuite, nous allons nous focaliser sur la possibilité que les emplois du futur ainsi isolés véhiculent des degrés de prévision spécifiques. Ce qui nous permettra, enfin, de déceler le rôle du futur simple français dans le discours de vulgarisation économique.

This contribution deals with enunciative conditions underlying the semantic values of French futur simple. In particular, the attention will be focussed on some cotextual and contextual elements which could influence the enunciative situation (Kerbrat-Orecchioni, 2002). The study of the uses of future simple will be carried out on the basis of a corpus of 2328 texts -643236 tokens - belonging to the economy section of Le Figaro ( $1^{\text {st }}$ January $-28^{\text {th }}$ February 2007). As economy language is based on predictions, the main aim will consist of the research of futur simple values expressing prediction. In addition, a particular focus will be given to the possibility that specific degrees of prediction might depend on futur simple values. Finally, this study will discover the role of French futur simple in economic popular discourse.

\section{INDEX}

Mots-clés : corpus de presse, discours économique, futur simple français, Situation énonciative, valeurs sémantiques

Keywords : economic discourse, Enunciative situation, French futur simple, press corpus, semantic values

\section{AUTEUR}

\section{ALIDA MARIA SILLETTI}

Università degli Studi di Modena e Reggio Emilia,Italie 